\title{
A PHOTOGRAPHIC METHOD OF DETECTING CHANGES IN A COMPLICATED GROUP OF OBJECTS
}

By M. H. Stillman

\section{CONTENTS}

I. Introduction Page

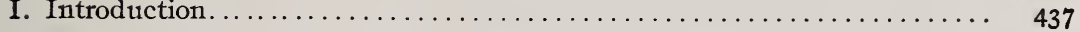

II. General description of method, with illustrations............... 437

III. Factors of importance in the successful use of the method ............ $43^{8}$

I. Photographic plates and development................. 438

2. Precautions in making the exposures................. 447

IV. Applicability of the method .......................... 448

\section{INTRODUCTION}

The method to be described has been used for some time in certain branches of physics ${ }^{1}$ and astronomy. ${ }^{2}$ It was recently independently devised by the writer and developed in cooperation with Dr. H. E. Ives, recently of the United States Air Service, for use in military operations.

The method is, however, applicable to a so much wider range of uses than the fields in which it has yet been employed that it seems desirable to describe the method and a few of its possible applications.

\section{GENERAL DESCRIPTION OF METHOD, WITH ILLUSTRATIONS}

The method may be described as follows: A negative is made of the group of objects in which a change is expected. After the change is supposed to have occurred a second negative is made with the same camera (or one of the same kind) on a plate of the same kind and from as nearly as possible the same position as used in making the first negative. A positive is printed from one of the negatives, is superposed upon the other negative so as to bring them into register, and the combination is viewed against a source of light. When the photographs are properly made,

\footnotetext{
${ }^{1}$ Hodgson and Wilsey, Communication No. 42 of Eastman Kodak Co. Research Laboratory, J. Op. Soc., 1, Nos. 2 and 3 ; 1917.

${ }^{2}$ Harvard College Observatory Circular No. 79, p. x.

I81067 $7^{\circ}-20$ 


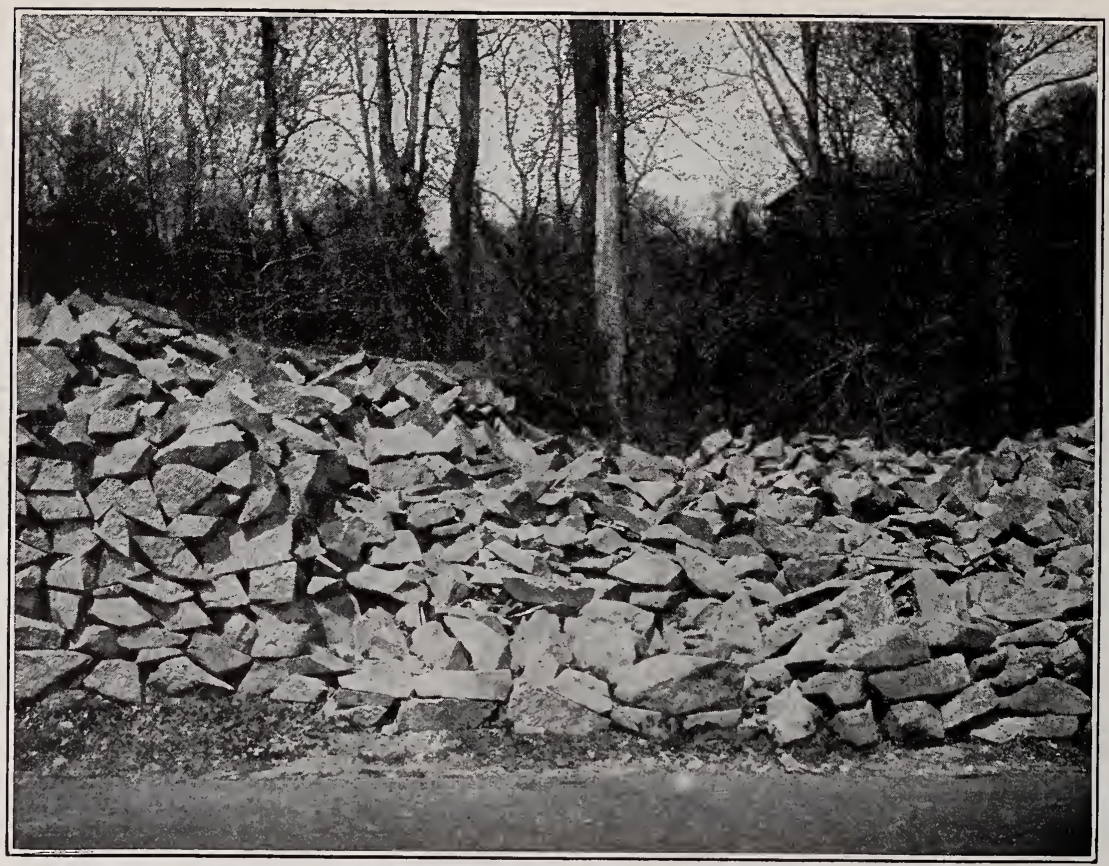

FIG. I.-First photograph. This illustrates the initial appearance of the pile of rocks

those parts of the combination which correspond to the unchanged portion of the group of objects will appear as a field of practically uniform density, while a change in the group will be revealed by a considerable departure from this uniform density.

This is illustrated in Figs. I to 7 , inclusive. Fig. I shows a pile of rocks before the change. Fig. 2 shows the same pile after making a few changes which, it will be noted, are not conspicuous. Fig. 3 shows the appearance when the second negative is superposed upon the positive of the first. Similarly, Figs. 4 to 7 , inclusive, show the application of the method to structural work. It usually makes little difference in the effectiveness of the method whether the positive is made from the first or second negative. These illustrations will be referred to later.

\section{FACTORS OF IMPORTANCE IN THE SUCCESSFUL USE OF THE METHOD}

\section{PHOTOGRAPHIC PLATES AND DEVELOPMENT}

Fig. 8 is a typical curve of the relation between the density and exposure of a photographic plate. 


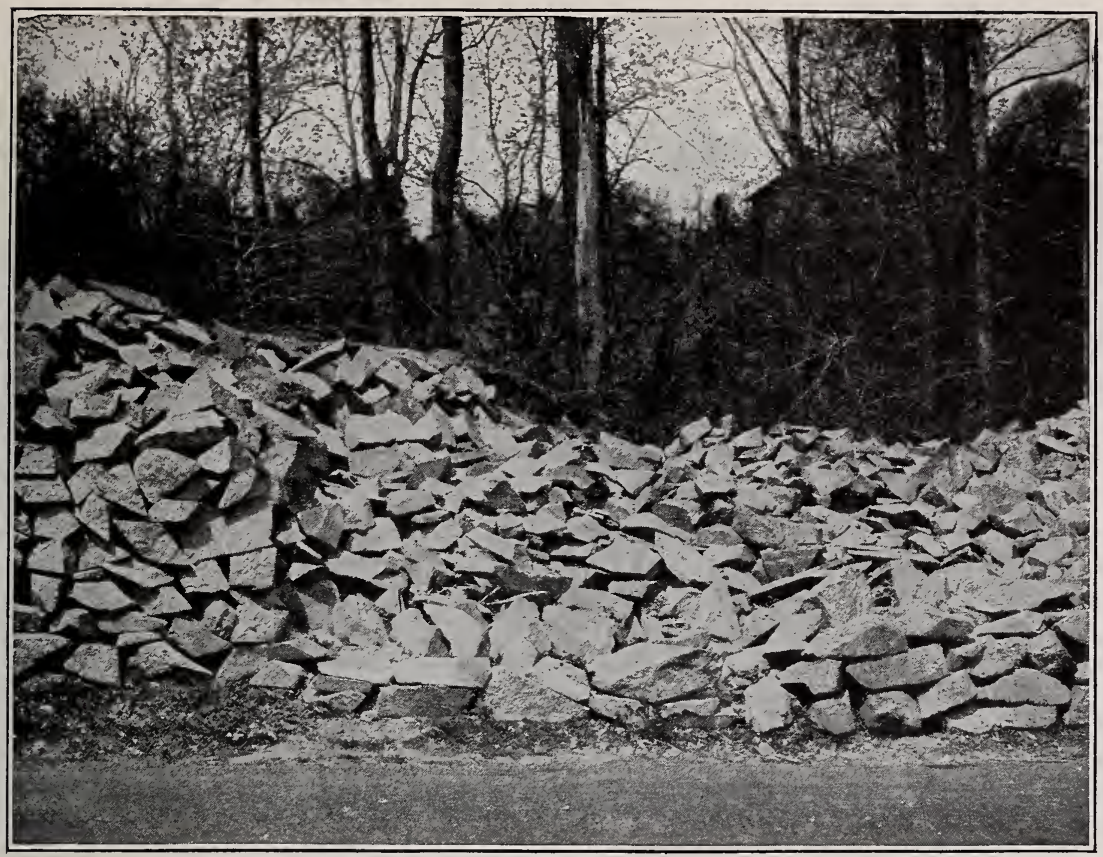

FIG. 2.- Second photograph, illustrating the appearance of the pile of rocks after a few changes in it have been made. Note the difficulty of detecting the changes and then refer to Fig 3.

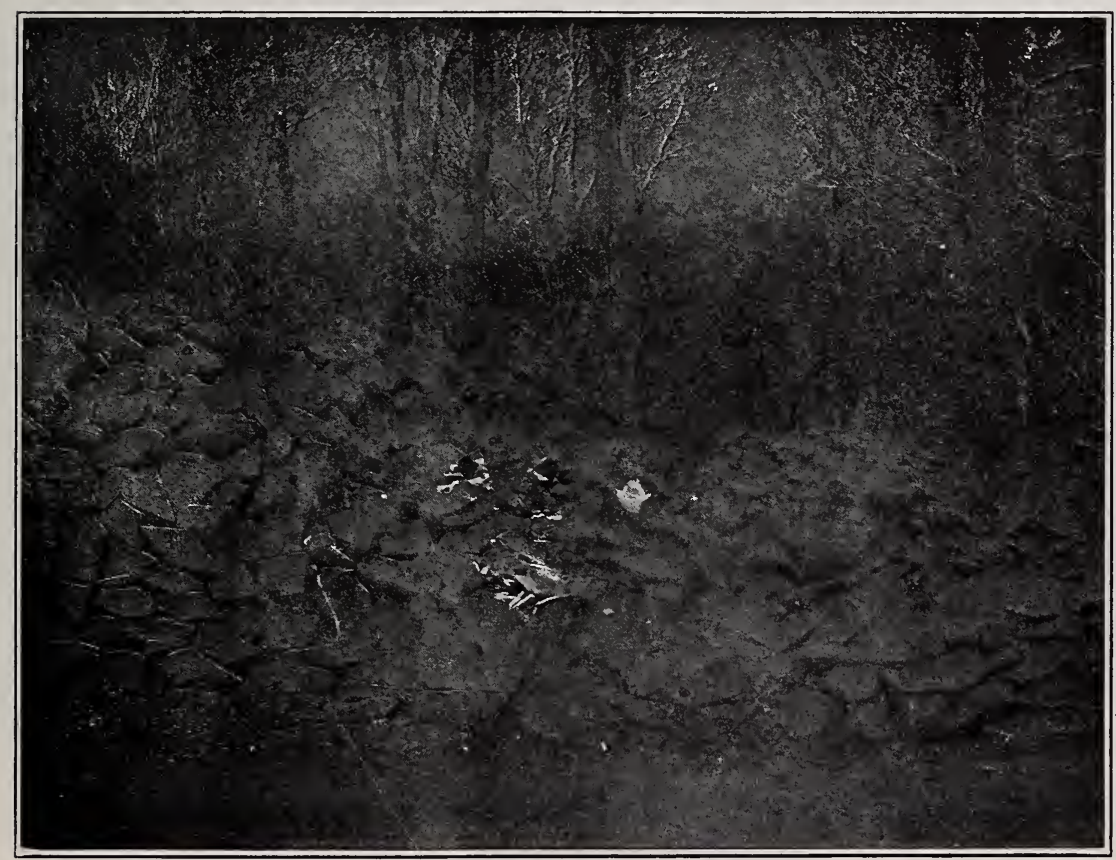

III. 3.-Superposition of negative of Fig. 2 and positive of Fig. I. The marked iariations in shade indicate the changes 

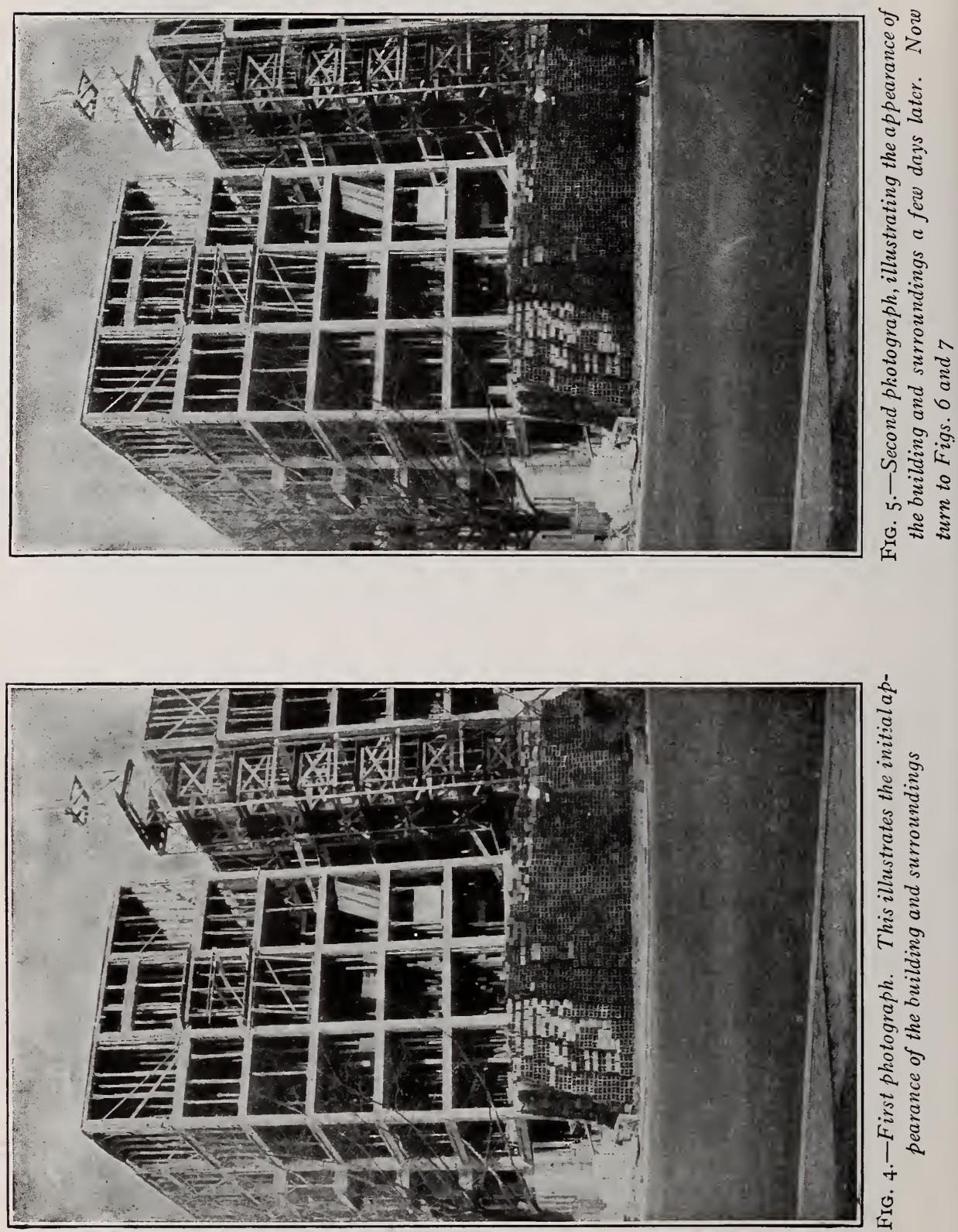


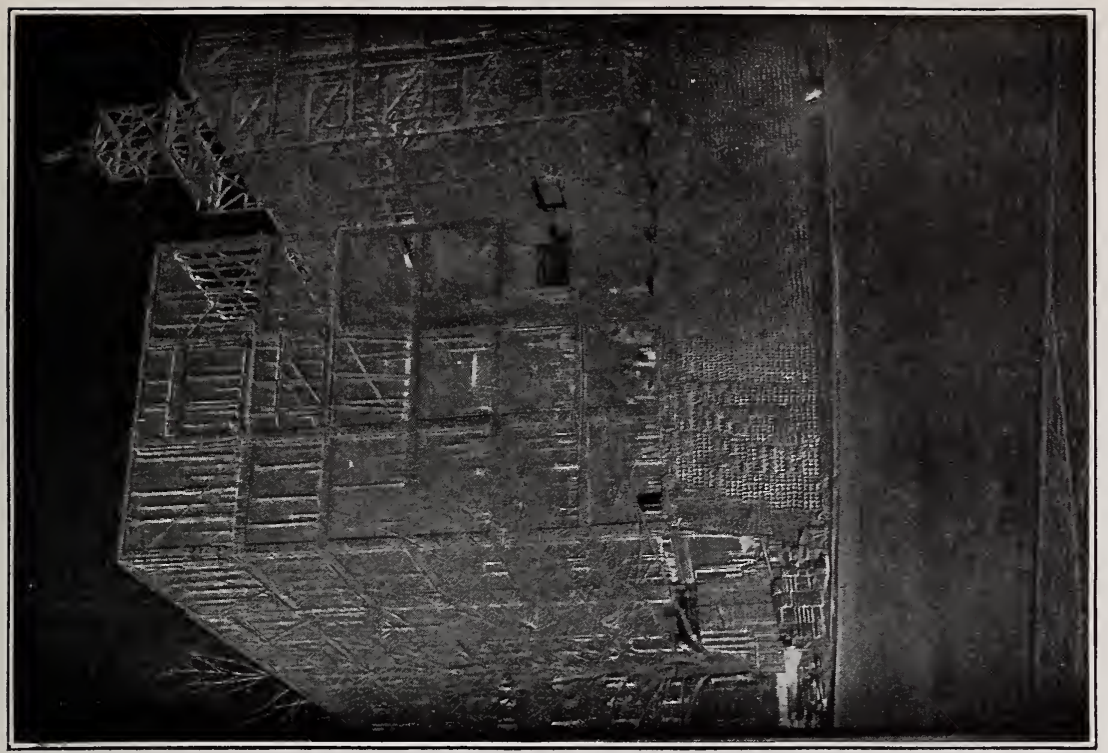

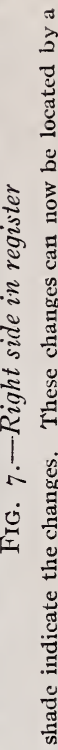

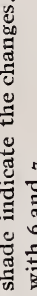

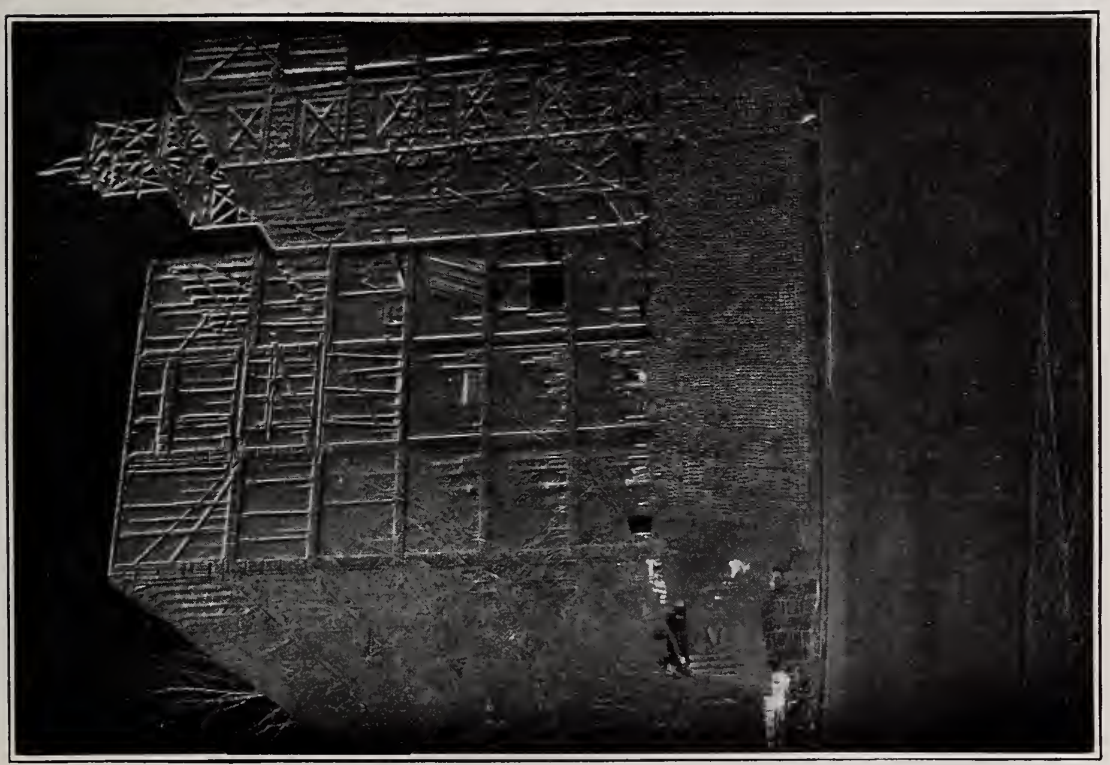

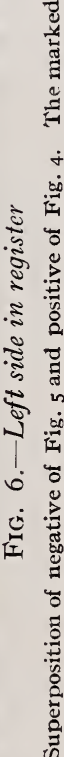


Letting $D$ represent the density, $E$ the exposure, $a$ the inertia, and $\gamma(=\tan \theta)$ the contrast, the equation of the straight-line portion of this curve is

or

$$
\frac{D}{\log E-\log a}=\gamma
$$

$$
D=\gamma(\log E-\log a) .
$$

The relation that must exist between the contrasts of the positive and the two negatives in order that the positive printed from one of the negatives when superposed in register upon the other negative will form a combination of uniform density, except in those parts corresponding to changes in the object, will now be derived. It will be assumed for the present that the exposures

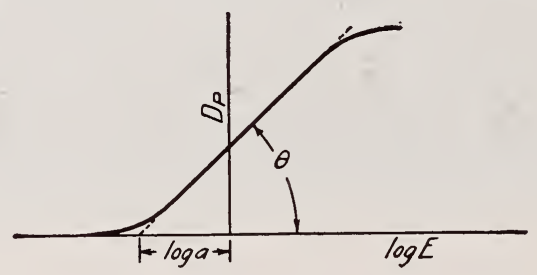

FIG. 8.

on all of the plates correspond to the straight-line portions of their characteristic curves.

Let $R \equiv$ reflecting power of the original object at any part.

$I_{1} \equiv$ illumination of object when exposing for the first negative.

$t_{1} \equiv$ time of exposure of the first negative.

$k_{1} \equiv$ fraction of the light reflected from the object which reaches the plate of the first negative.

$D_{1} \equiv$ density of first negative at part corresponding to that part of original object having a reflecting power of $R$.

$\gamma_{1} \equiv$ contrast to which first negative is developed.

$a_{1} \equiv$ inertia of first negative.

$I_{2} \equiv$ illumination of object when exposing for the second negative.

$t_{2} \equiv$ time of exposure of the second negative.

$k_{2} \equiv$ fraction of the light reflected from the object which reaches the plate of the second negative.

$D_{2} \equiv$ density of second negative at part corresponding to that part of the original object having a reflecting power of $R$.

$\gamma_{2} \equiv$ contrast to which second negative is developed. 
$a_{2} \equiv$ inertia of second negative.

$I_{\mathrm{p}} \equiv$ illumination of the second negative when printing the positive.

$t_{\mathrm{p}} \equiv$ time of exposure of the positive.

$D_{\mathrm{p}} \equiv$ density of positive at part corresponding to that part of original obiect having a reflecting power of $R$.

$\gamma_{\mathrm{p}} \equiv$ contrast to which positive is developed.

$a_{\mathrm{p}} \equiv$ inertia of positive.

The light reflected from that part of the object of reflecting power $R$ when the illumination is $I_{1}$ is $I_{1} R$. Then the exposure of the plate for the first negative may be expressed as $k_{1} R I_{1} t_{1}$, where $k_{1}$ is the fraction of the light reflected from the object which reaches the plate. Substituting this expression for $E$ in equation (I) and using nomenclature given above, the density of the first negative at the part corresponding to the part of object having the reflecting power of $R$ is given by

$$
D_{1}=\gamma_{1}\left[\log k_{1} I_{1} t_{1}+\log R-\log a_{1}\right],
$$

and similarly, the density of the corresponding part of the second negative when the exposure incident on the object is $I_{2}$ is given by

$$
D_{2}=\gamma_{2}\left[\log k_{2} I_{2} t_{2}+\log R-\log a_{2}\right] .
$$

Suppose that the positive is printed from the second negative. Then the density of this positive at the part corresponding to that part of the original object having the reflecting power of $R$ is given by

$$
D_{\mathrm{p}}=\gamma_{\mathrm{p}}\left[\log I_{\mathrm{p}} t_{\mathrm{p}}-\left(D_{2}+\log a_{\mathrm{p}}\right)\right] .
$$

From (3) and (4)

$$
D_{\mathrm{p}}=\gamma_{\mathrm{p}}\left[\log I_{\mathrm{p}} t_{\mathrm{p}}-\left(\gamma_{2}\left\{\log k_{2} I_{2} t_{2}+\log R-\log a_{2}\right\}+\log a_{\mathrm{p}}\right)\right] .
$$

Now it is required that this positive from the second negative, when superposed upon the first negative, shall form a combination of uniform density except at those parts corresponding to changes in the group of objects between the exposures of the first and second negatives; that is:

$$
D_{1}+D_{\mathrm{p}}=K
$$

where $K$ is the uniform density of the combination. Substituting into (6) the values of $D_{1}$ and $D_{\mathrm{p}}$ from (2) and (5),

$\gamma_{1}\left[\log k_{1} I_{1} t_{1}+\log R-\log a_{1}\right]+\gamma_{\mathrm{p}}\left[\log I_{\mathrm{p}} t_{\mathrm{p}}-\left(\gamma_{2}\left\{\log k_{2} I_{2} t_{2}+\log R-\right.\right.\right.$

$$
\left.\left.\left.\log a_{2}\right\}+\log a_{\mathrm{p}}\right)\right]=K \text {. }
$$


Differentiating (7) with respect to $R$, and solving for $\gamma_{\mathrm{p}}$ gives

$$
\gamma_{p}=\frac{\gamma_{1}}{\gamma_{2}}
$$

Thus the condition for securing correct results is that the contrast of the positive equals the ratio of the contrast of the negative which is superposed upon the positive to the contrast of the negative from which the positive is printed.

It is evidently desirable that both the positive and its superposed negative have considerable contrast in order to render as conspicuous as possible any changes that may have occurred in the group of objects. This, it will be noted, is entirely consistent with the necessary relations between the contrasts as expressed in (8).

As a corollary of (8), a relation of importance in the practical application of the method may now be noted. In order to secure the correct relation between the positive and its superposed negative, the negative from which the positive is to be printed may be developed to a contrast of unity, and then the positive and other negative developed together or separately to secure equal contrasts in the two, or the two negatives may be developed together or separately to equal contrasts, and the positive then developed to a contrast of unity. In the present state of the art of photography, those using the method only occasionally would probably not make use of this relation, but would by trial develop the positive to a contrast sufficiently near to $\frac{\gamma_{1}}{\gamma_{2}}$ to secure the desired results. For extensive use, however, it would probably be found well worth while to standardize a developer and method of using it which would give a contrast of approximately unity, and also, in order to secure the large differences in density desired in the superposed positive and negative, another development which would give a greater contrast.

It will be noted that the only limitations imposed upon the values of the exposures of the two negatives and the positive is that they shall correspond to the straight-line portions of the characteristic curves. Thus there will often be some latitude allowable in the choice of the exposures, the use of the larger allowable values of $I_{1} t_{1}$ and $I_{\mathrm{p}} t_{\mathrm{p}}$ resulting in a combination of positive and negative of greater density than when the smaller allowable values are used.

Inasmuch as a given difference in density (= ratio of opacity) is more conspicuous in a field of small density than one of greater 
density, it is desirable to use the smallest allowable exposures in making the positive and the negative that is superposed upon it in order to reduce to the minimum, consistent with the contrast, the density of the combination of positive and negative. Even so, the combined density will in most cases be considerable if, as is recommended, both the positive and negative have considerable contrast.

A graphical representation of the relations between the exposures and densities of the positive and negatives will not only

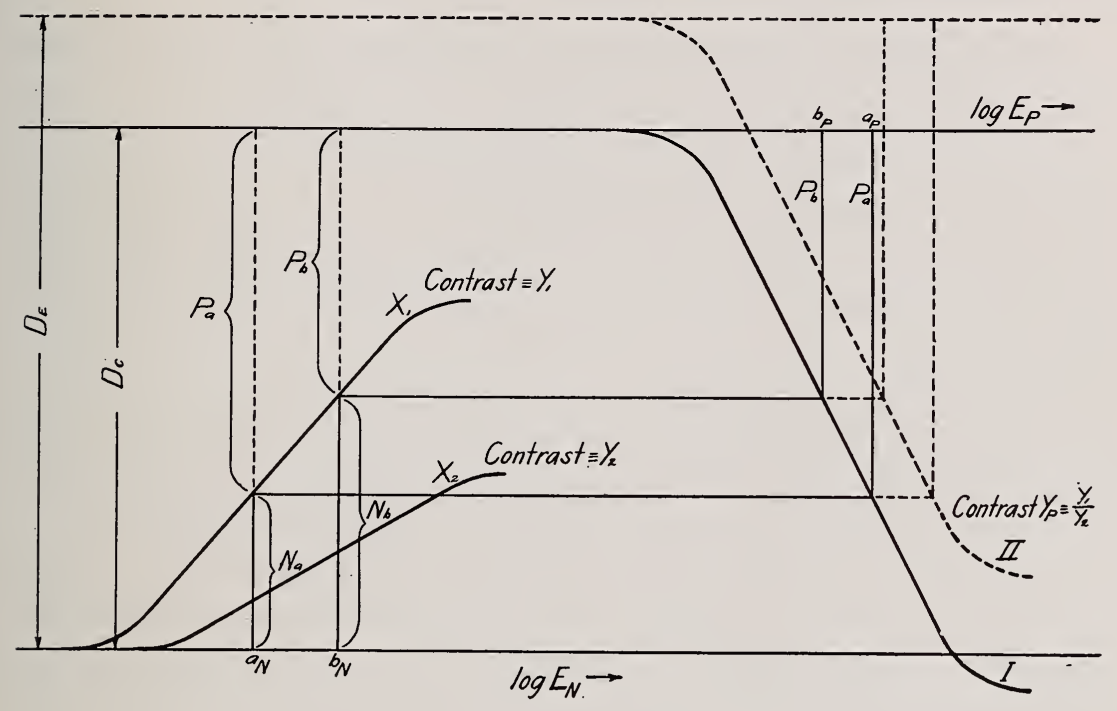

Fig. 9

aid in the further discussion, but will illustrate that which has preceded.

In Fig. 9 the curves at the left are the characteristic curves of the negatives, $X_{1}$ being the curve of the first negative, which it will be assumed is superposed upon the positive, while $X_{2}$ is that of the second negative, from which the positive is printed. The curves at the right are the inverted curves of the positive, curve II being curve I shifted vertically.

The first negative (curve $X_{1}$ ) has been developed to a contrast of $\gamma_{1}$. Suppose that two different portions of the negative had been given exposures, the logarithms of which are $a_{\mathrm{N}}$ and $b_{\mathrm{N}}$, the resulting densities are then $N_{\mathrm{a}}$ and $N_{\mathrm{b}}$, respectively. It is desired to superpose upon this negative a positive of such densities $P_{\mathrm{a}}$ and $P_{\mathrm{b}}$, corresponding to $N_{\mathrm{a}}$ and $N_{\mathrm{b}}$, that a combination or uniform density $D_{\mathrm{c}}$ will be produced; that is, $P_{\mathrm{a}}+N_{\mathrm{a}}=P_{\mathrm{b}}+$ 
$N_{\mathrm{b}}=D_{\mathrm{c}}$. Then $P_{\mathrm{a}}-P_{\mathrm{b}}=N_{\mathrm{b}}-N_{\mathrm{a}}$. Thus the difference in density between two given parts of the positive must be the same as the difference in density between corresponding parts of the negative, but in the reverse order. It has already been shown that in order to obtain from the second negative (curve $X_{2}$ ) a positive that will fulfill these conditions this positive must be so developed that its contrast $\gamma_{p}$ is given by $\gamma_{p}=\frac{\gamma_{1}}{\gamma_{2}}$. The inverted characteristic curves, I and II, of the positive are therefore shown with this $\gamma_{p}$.

If the positive plate is given an exposure which would result in the densities indicated on curve I, the resulting density of the combination of positive and negative will evidently be $D_{\mathrm{c}}$. If the exposure is increased, but not sufficiently to bring the exposure of any part of the positive outside of the straight-line portion of its curve, densities such as indicated in curve II will result, and the density of superposed positive and negative will be $D_{\mathrm{E}}$ instead of $D_{\mathrm{c}}$.

If both the first and second negatives were made with the same kind of plate, given the same exposure and developed to the same contrast, the contrast $\gamma_{p}$ of the positive would be made unity and it would not be necessary to confine the exposures of the negatives to the straight-line portions of their curves in order to obtain the correct density relations between the positive and negative. It is in general impracticable to realize all of these conditions in practice, and it is therefore desirable to confine the exposures to the straight-line portions. This makes it necessary to select plates of which the latitude is sufficient to accommodate the range of exposures that will be involved.

The graphical construction to show the exposures on the positive plate corresponding to those used in producing the negatives is evident from Fig. 9. It is interesting to notice that although the difference in the densities of any two parts of the positive is the same as the difference in densities of the corresponding parts of the negative upon which it is superposed, the ratio of exposures used in producing these equal differences of density is in general not the same. This is immediately evident upon comparing in Fig. 9 the difference between the logarithms of the exposures $a_{\mathrm{N}}$ and $b_{\mathrm{N}}$ of the negative with the corresponding difference between the logarithms of $a_{\mathrm{P}}$ and $b_{\mathrm{P}}$ of the positive. Using the nomenclature of Fig. 9, and by the application of the equations of the straightline portions of the characteristic curves of the positive and 
negative, the relation between the ratios of exposures is shown to be

$$
\left(\frac{a_{\mathrm{N}}}{b_{\mathrm{N}}}\right)^{\gamma_{1}}=\left(\frac{b_{\mathrm{P}}}{a_{\mathrm{P}}}\right)^{\gamma_{\mathrm{p}}}
$$

Thus the ratio of exposures is not the same un.ess $\gamma_{1}=\gamma_{p}$, but by (8), $\gamma_{\mathbf{p}}=\frac{\gamma_{1}}{\gamma_{2}}$

Hence the condition under which the ratio of exposures for corresponding parts of the positive and superposed negative are the same is that

so that $\gamma_{2}=\mathrm{I}$.

$$
\gamma_{1}=\frac{\gamma_{1}}{\gamma_{2}}
$$

While exact uniformity in density of all portions of the superposed positive and negative, not corresponding to changes in the group of objects, has been the basis of the above discussion, this ideal condition can be only approximated in practice. Thus, referring to Figs. 6 and 7 , it will be observed that the sky and the white sign on the building appear considerably darker than the remainder of the unchanged portions of the picture. This is due principally to the fact that the positive was not developed quite to the contrast of unity. If the development had been carried further to increase the contrast, $\gamma_{p}$, a degree of uniformity in density similar or superior to that shown in Fig. 3 would have been obtained. Such departures from complete uniformity are ordinarily not a limitation of the value of the method but, on the contrary, permit the observer to locate with respect to the various parts of the picture the changes indicated by the marked differences in density.

\section{PRECAUTIONS IN MAKING THE EXPOSURES}

In exposing for the negatives it is desirable that the camera be, as nearly as possible, in the same position for both exposures. This is especially important when both near and distant objects are included in the picture. When all parts of the picture are in sensibly the same plane, as when a photograph is taken from high in the air, the position of exposure may vary considerably and good results still be obtained.

If the two negatives are made from the same general direction but at different distances from the object, one of these may be enlarged or reduced as necessary, or it may be projected at the proper size onto a ground-class screen and its image matched there with the positive, or vice versa. When the negative and positive are not of the same size, it will of course be impossible to bring 
them into exact register, but if they are of nearly the same size it will be found that by shifting them slightly with respect to each other one portion after another will approximately register, permitting the entire picture to be examined by sections. Such shifting is illustrated in Figs. 6 and 7.

The light conditions at the time of making the two exposures should be as nearly as possible the same as regards position of source of light, intensity, and quality. It is evident, for example, that if one picture of a landscape were taken in the morning and another in the afternoon the changed position of the shadows would show up so prominently in the result that the changes sought might be overlooked.

\section{APPLICABILITY OF THE METHOD}

The method is evidently applicable in military operations. Exposures made from practically the same position at successive periods of time give a record of changes which could easily be detected by this method of superposition. Newly erected camouflage and other military work of the enemy would be revealed.

An interesting application of the method would be its use in discovering the introduction or removal of an object, such as a battery, from behind a protecting screen which would allow so little direct vision as to absolutely preclude the possibility of the object being detected by direct observation. By eliminating the confusing details of the screen this method would permit the object to be detected and its general shape determined.

In certain types of engineering work progress can be very well shown by an application of this method. For example, in the case of a bridge or other framework under construction provision is easily made for taking a photograph from the same position at frequent intervals of time. Then by superposing the proper positive and negative the progress in any period would be revealed without painstaking comparisons.

Changes in complicated documents, drawings, and engravings are brought out clearly by this method. This suggests its application to the verification of the copies of engravings, drawings, and the like.

While the best results can undoubtedly be secured by a skilled operator giving attention to all the factors discussed herein, yet the method can be used successfully in many instances by those having only a very limited knowledge of photography and by the use of comparatively inexpensive apparatus.

Washington, December I, I9I9. 


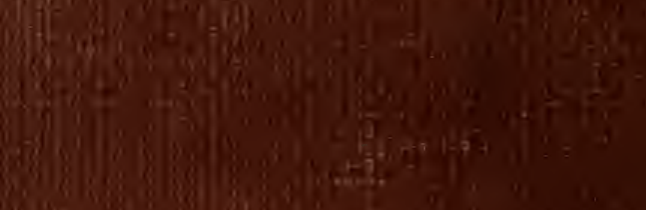


